\title{
Cost and Clinical Efficacy of Laparoscopic vs. Open Approach in Liver Resection
}

\section{Subash Sharma, Yingjian Liang, Guo Xiao, Wang Hang, Hui Peng and Lianxin Liu*}

Key Laboratory of Hepatosplenic Surgery, Ministry of Education, Department of General Surgery, The First Affiliated Hospital of Harbin Medical University, Harbin, China

"Corresponding author: Lianxin Liu, Key Laboratory of Hepatosplenic Surgery, Ministry of Education, Department of General Surgery, The First Affiliated Hospital of Harbin Medical University, Harbin, China, Tel: 8645185553886; Fax: 8645153670428; E-mail: liulianxin@ems.hrbmu.edu.cn

Received date: August 13, 2018; Accepted date: August 30, 2018; Published date: September 6, 2018

Copyright: ( 2018 Sharma S, et al. This is an open-access article distributed under the terms of the Creative Commons Attribution License, which permits unrestricted use, distribution and reproduction in any medium, provided the original author and source are credited.

\begin{abstract}
Background: Hesitation but gradual recognition of laparoscopic liver resection has been gaining popularity in developed countries among the specialized hospitals. On contrary, there has always been a debate about high cost and complexity of laparoscopic as compared to open liver resection resulting in the delayed adoption of laparoscopic approach in many centers.

Methods: We retrospectively reviewed medical records of all patients at the $6^{\text {th }}$ department (Hepatobiliary Unit) of the $1^{\text {st }}$ affiliated hospital of Harbin Medical University who underwent elective LLR $(n=102)$ or OLR $(n=198)$ from January $1^{\text {st }}, 2014$ to November $1^{\text {st }}, 2017$. Patient demographics, diagnosis, operation type, operative duration, estimated blood loss, blood transfusion requirement pathology, length of hospital stay, ICU stays and complications were compared between laparoscopic and open approach. Hospital billing data were collected for each case to determine the mean hospital costs per case.
\end{abstract}

Conclusion: Our study justifies that LLR is safe with remarkably better clinical benefits as well cost advantage to some extent. Short operating duration, short hospital stay, lower chances for ICU need, less blood loss, less blood transfusion requirement, and low occurrence of complications are benefits offered by the laparoscopic procedure.

Keywords: Laparoscopic liver resection (LLR); Open liver resection (OLR); Space occupying lesion (SOL); Clinical efficacy; Complications

Abbreviations: LLR: Laparoscopic liver resection; OLR: Open liver resection; SOL: Space-occupying lesion; ICU: Intensive care unit; HMU: Harbin medical university; COPD: Chronic obstructive pulmonary disease; HCC: Hepatocellular carcinoma.

\section{Introduction}

Liver resection is the surgical resection (removal of all or part) of the liver. An indication of liver resection includes hepatic neoplasm, intrahepatic gallstones, and parasitic cysts of liver hepatic trauma as well as liver transplantation. Hepatic neoplasm could be benign or malignant. Benign includes hepatocellular adenoma, hepatic hemangioma, and focal nodular hyperplasia. A malignant neoplasm, on the other hand, includes HCC and metastases from colorectal cancer. Liver resection remains the only potentially curative treatment for primary and metastatic liver tumors.

The first liver resection was reported by Dr. Ichio Honjo (1913-1987) of (Kyoto University in 1949, and Dr. Jean-Louis LortatJacob (1908-1992) of France in 1952 [1,2]. The first laparoscopic nonanatomic resection of a Focal Nodular Hyperplasia (FNH) was reported by Gagner and colleagues in 1992, and this was followed by the first report of laparoscopic anatomic liver resection in 1996 [3]. Laparoscopic liver surgery was slower to develop than other fields of laparoscopic surgery because of difficult anatomy, the complexity of procedure and fear of uncontrolled bleeding or gas embolism. However, Laparoscopic Liver Resection (LLR) is associated with significant advantages: faster recovery, less post-operative pain, less morbidity, easier subsequent surgery and better cosmetic results. With the improvement in surgical technique, anesthetic drugs, and intensive care it became possible to perform complex liver resections with very low morbidity and mortality. Open as well as laparoscopic both approaches are practiced for liver resection these days. Even though OLR hasn't lost its important place, LLR has undergone a steady increase in these few years. The liver is divided into VIII anatomical segments.

Different classifications are used to define liver resections according to the segment or segments resected. The most widely used system of nomenclature of the liver is couinaud, 1957. This classification names the liver resection as i) V-VIII; right hepatectomy ii) IV-VII; right lobectomy iii) II-IV; left hepatectomy iv) II-III; left lobectomy v) II, III, IV, V, VIII; extended left hepatectomy. The postoperative and oncologic outcome of LLR has been proven to be as effective as with traditional OLR when performed by experienced surgeons. Laparoscopy seems to be more widely performed for wedge resections and other minor liver resections since major resections can be very challenging and left only in the hands of a few experts. OLR was also widely used for any kind of hepatic lesions. In some highly specialized centers, even some robotic surgeries were also performed beside LLR and OLR. Different authors had different views in terms of cost but in terms of clinical benefits, almost all authors were in favor of laparoscopic approach. Excessive blood loss is associated with increased perioperative morbidity.

Laparoscopic liver resection has been gaining popularity more and more these days. Over this decade feasibility of LLR has been improved rapidly as training, experience and facility for laparoscopic techniques 
have increased. LLR has leaped forward from the application in peripherally located benign lesions to large and malignant lesions. With the development of the society, the need for medical services has been increasing day by day. The advancement of diagnostic tools has aided opportunities in the choice of operation. Minimally invasive laparoscopic liver resections; therefore, has become more accurate and feasible. Universally accepted advantages of LLR includes minimal trauma, less pain as well as quick recovery. Long-term complications of surgery such as incisional hernias and differences in outcomes such as persisting pain would be other advantages of LLR over OLR.

The curative effect, clinical benefits as well as the cost of operation has always been debated. Cost of medical equipment has always been higher for LLR as they would require expensive disposable instruments, as well as medical equipment (e.g. Laparoscope). Therefore, our future goal is to decrease the overall cost by increasing the clinical efficacy as well as reducing the medical costs.

\section{Materials and Methods}

After getting permission from the department, a computer database was used to retrospectively collect all data from January 2014 to November 2017. Our goal was to collect about 300 randomized cases retrospectively from the computer database and then use statistical software to analyze those data. These data included cases that underwent liver resection in the $6^{\text {th }}$ department of the $1^{\text {st }}$ affiliated hospital of HMU (hepatobiliary surgery) for any kind of SOL in the liver. A patient who has already undergone chemotherapy and other simultaneous non-hepatic surgery for bulky lesions infiltrating the diaphragm, liver cysts and vascular encasement were excluded. We excluded three cases that were converted from laparoscopic to open approach because of such low number of conversions.

We began our research with 102 (LLR) and 198 (OLR) patients. The total cost of treatment of each patient was collected from the computer database. This cost included all expenses that occurred from admission to discharge of the patient. Therefore total cost included inpatient cost, medicine cost, and operation theatre cost, anesthesia cost, nursing cost as well as ICU or ventilator cost and miscellaneous according to the need of the patient. We just collected value of total cost and didn't collect the cost of individual variables. All the cost mentioned in this article is in Chinese RMB (as of $10^{\text {th }}$ May, 2018; $100 \mathrm{RMB}=15.78$ USD). Per day inpatient cost in general ward was $50 \mathrm{RMB}$ (without any drugs or nursing cost) while that in ICU was 610 RMB (without drugs, nursing charges or ventilator charges as shown by billing data records). There were no strict criteria directing operative approach (LLR vs. OLR) but instead was decided by each individual operating surgeon.

OLR cases were operated using either upper midline incision above the umbilicus or a long chevron incision. Pringle maneuver was sometimes used in open resection (20 minutes) in the cases where there was excessive bleeding.

Tiny incisions trocars laparoscopic instruments are used to operate the cases of LLR. The operation begins with the use of peri-umbilical gel port hereby entering the abdominal cavity under direct visualization using optiview (Ethicon Endosurgery, Inc.) trocar accompanied by placement of remaining trocars. Laparoscopic intraoperative ultrasound is occasionally used to evaluate the hepatic SOL. Ultrasonic equipment is used to precisely dissect the liver parenchyma tissue and major blood vessels with staplers. Majority of operations were performed using pure laparoscopic approach and a few as hand assisted.
Different kinds of operations are performed according to the segments involved. Our cases included partial hepatectomy, left lateral lobectomy, left lobe resection, resection of right hepatic trisegmentectomy and right hemi-hepatectomy. The resected tissue is finally removed via the hand port and hemostasis is achieved with cautery, suture, and/or hemostatic agents according to the intensity of the bleeding. Pringle maneuver was rarely used for cases in laparoscopic approach.

Operative duration, the volume of intra-operative blood loss, the volume of intra-operative blood transfusion, duration of hospital stay, duration of ICU stay and occurrence of complications were the chosen variables to analyze for clinical efficacy. Clavien-dindo system was used for the grading of surgical complications.

The research applies clinical patient-matched cohort statistical methods. Statistical analysis was performed using SPSS 22.0 software and the GraphPad Prism software package (v. 5.01). All data are presented as means \pm SD. Variance between two groups was analyzed using unpaired t-tests. $\mathrm{P}<0.05$ was considered statistically significant. The collected data was managed in Microsoft Excel and histograms and pie chart was merged using adobe illustrator.

\section{Results}

The results were calculated by SPSS 22.0 statistical software. There was a total sample size of 300 patients, which included 102 (LLR) and 198 (OLR) patients. The result of two-tailed unpaired t-tests revealed that choice of operative approach (LLR/OLR) didn't have any statistical significance in terms of cost $(\mathrm{P}=0.4231)$. The mean $\mathrm{SD}$ of the total cost for LLR group was RMB $58740 \pm 1585(\mathrm{~N}=102)$ while that with OLR was RMB $61110 \pm 1964(\mathrm{~N}=198)$. Hence, the mean total cost of LLR group was found slightly lower compared to OLR group. The variations of cost (SD) in LLR group RMB $( \pm 1585)$ were also slightly lower than OLR group RMB $( \pm 1964)$. The average age in LLR group was $48.94 \pm$ 1.219 years while that in OLR group was $52.38 \pm 0.0805$ years $(\mathrm{P}=0.0157)$. In both groups (LLR or OLR) benign cases were higher than malignant cases as shown in the table (Table 1 and Figure 1).

\begin{tabular}{|c|c|c|c|c|}
\hline Variable & $\operatorname{LLR}(\mathrm{N}=102)$ & OLR ( $N=198)$ & t-test & $\begin{array}{l}P \\
\text { value }\end{array}$ \\
\hline Total Liver Resections & 102 & 198 & & \\
\hline Sex: Male & 56 & 115 & & \multirow{2}{*}{0.5983} \\
\hline Female & 46 & 83 & & \\
\hline Age (years) (Mean SD) & $48.94 \pm 1.219$ & $52.38 \pm 0.8005$ & & 0.0157 \\
\hline \multicolumn{3}{|l|}{ Disease Species: } & & \\
\hline Benign $(N=198)$ & 70 & 128 & & \\
\hline Malignant( $\mathrm{N}=102)$ & 32 & 70 & & \\
\hline Cost (Mean SD) & $58740 \pm 1585$ & $61110 \pm 1964$ & $\mathrm{t}=0.8021$ & 0.4231 \\
\hline
\end{tabular}

Table 1: Patient demographics /general information. 
a

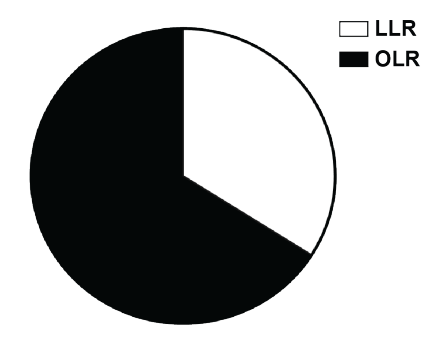

C

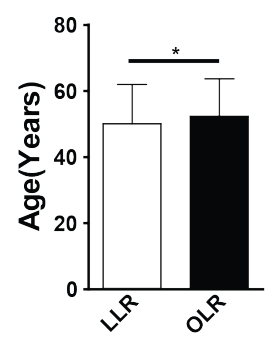

b

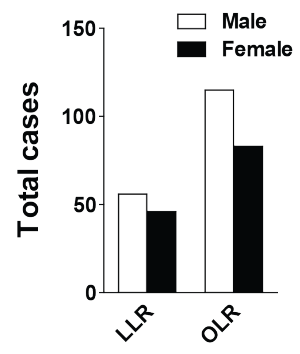

d

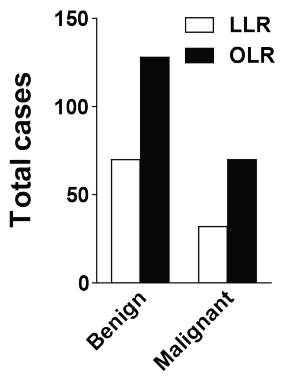

Figure1: (a) Distribution of patients for liver resection. (b) Distribution of liver resections by sex. (C) Distribution of liver resections by age. (d) Distribution of disease species (benign/ malignant) in Liver resection. The age is expressed as mean \pm SD. ${ }^{*} \mathrm{P}<0.05$.

The results of two-tailed unpaired t-tests showed that clinical benefits were positively associated with the selection of approach for liver resection (LLR/OLR). Moreover, two-tailed unpaired t-test revealed that LLR were positively associated with shorter operative duration $(\mathrm{P}=0.0002)$, lower intraoperative blood loss $(\mathrm{P}<0.0001)$, lower intraoperative blood transfusion $(\mathrm{P}<0.0001)$, shorter hospital stay $(\mathrm{P}<0.0001)$, shorter ICU stay $(\mathrm{P}=0.0002)$ and fewer complications $(\mathrm{P}=0.0266)$. Operation duration for LLR $214.5 \pm 8.078$ minutes was significantly less compared to OLR $250.0 \pm 5.261$ minutes. The average operative blood loss of LLR $194.7 \pm 25.76 \mathrm{ml}$ was remarkably lower compared to OLR $398.9 \pm 26.41 \mathrm{ml}$.

Meanwhile, the average intra-operative blood transfusion ( $\mathrm{ml}$ ) for LLR 167.6 $\pm 24.58(\mathrm{~N}=102)$ was lower compared to OLR $423.6 \pm 30.85$ $(\mathrm{N}=198)$. Average hospital stay for LLR group was $11.42 \pm 0.6191$ days and for OLR group was $15.62 \pm 0.6572$ days. Average ICU stay for LLR was $0.08824 \pm 0.03147$ and that for OLR was $0.3182 \pm 0.04017$. Per day inpatient stay cost $50 \mathrm{RMB}$ was much less compared to per day ICU cost of $610 \mathrm{RMB}$.

Post-operative complications were not encountered in 91 patients in LLR group and 160 patients in OLR group. Few patients in both groups presented with complications of fever $(L L R=9, O L R=19)$ and pleural effusion ( $L L R=1, O L R=4)$. Pneumonia, seroperitoneum dyspnea, ascites, CBD stenosis and renal injury were also other post-operative complications observed in patients in OLR group. LLR had an absence of post-operative complications like dyspnea, pneumonia, ascites/ abdominal distention, CBD stenosis, renal injury, sero-peritoneum (Figure 2).

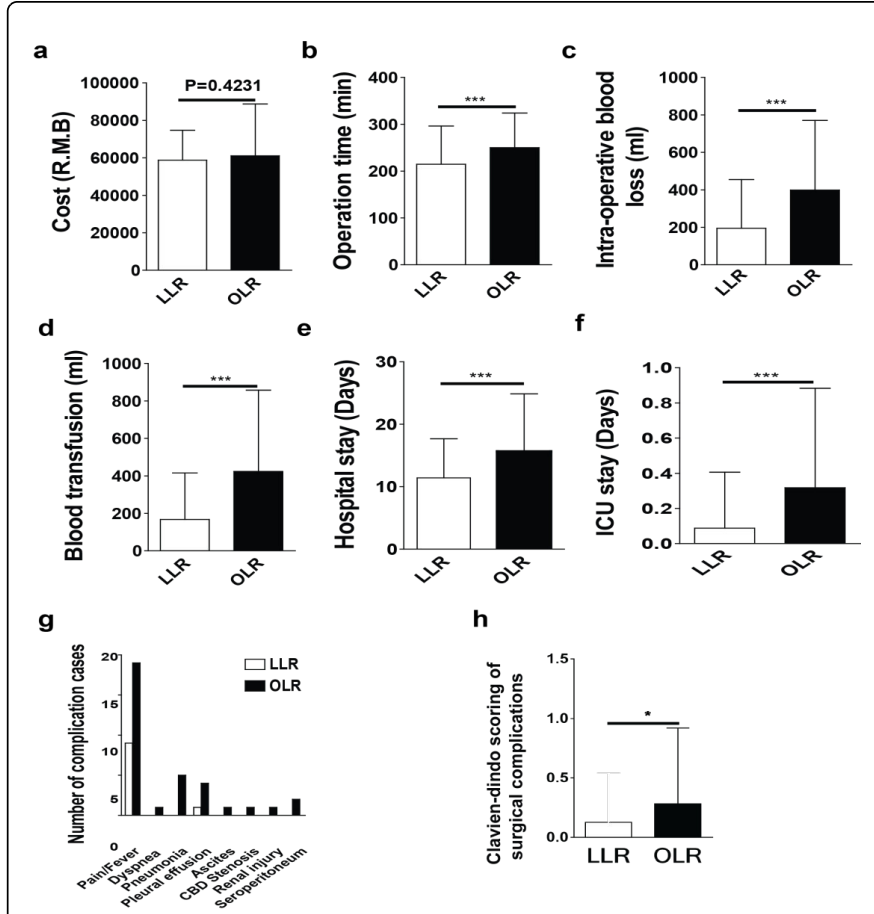

Figure 2: (a) The total hospital cost of liver resection. (b) The duration of the operation. (c) The volume of intra-operative blood loss. (d) The volume of blood transfusion. (e) The total duration of hospital stay. (f) The total duration of ICU stay. (g) A total number of post-operative complication cases. (h) Clavien-dindo scoring of surgical complications. The data are expressed as mean $\pm \mathrm{SD}$. ${ }^{*} \mathrm{P}<0.05 ;{ }^{* *} \mathrm{P}<0.001$.

Clavian-dindo classification was used to score the post-operative complications and the results were analyzed using two-tailed unpaired t-test which revealed that the complications scoring were lower in LLR with statistical significance $(\mathrm{P}=0.0266)$. The Mean SD of Clavien-dindo scoring for LLR was $0.1275 \pm 0.04103(\mathrm{~N}=102)$ while that for OLR was $0.2828 \pm 0.04533(\mathrm{~N}=198)$ (Table 2).

\begin{tabular}{|l|l|l|l|l|}
\hline Variable & LLR & OLR & $\mathbf{X}^{2}$ test & P-value \\
\hline Pain /Fever & 9 & 19 & 0.0474 & 0.8275 \\
\hline Dyspnea & 0 & 1 & 0.5168 & 0.4721 \\
\hline Pneumonia & 0 & 5 & 2.6194 & 0.1055 \\
\hline Pleural effusion & 1 & 4 & 0.4441 & 0.5051 \\
\hline Ascites /Abdominal distention & 0 & 1 & 0.5168 & 0.4721 \\
\hline CBD Stenosis & 0 & 1 & 0.5168 & 0.4721 \\
\hline Renal injury & 0 & 1 & 0.5168 & 0.4721 \\
\hline Seroperitonium & 0 & 2 & 1.0372 & 0.3084 \\
\hline
\end{tabular}

Table 2: Post-operative complications. 


\section{Discussion}

Increasing choice of LLR day by day indicates evidence that LLR is not only feasible and less invasive but also safe economically maintaining equal/more clinical efficiency. A variety of study methodologies are used with the majority of studies being retrospective case series or case-matched series. Some other methods being used are multicenter prospective study, case-cohort matched study; deviation based cost modeling and weighted average median cost, and value-based analysis.

In fact, Bhojani, et al. had evaluated all LLRs from 2006-2010 performed in their institution and each case was retrospectively matched to 2 open cases for a number of segments removed, patient's age and liver histology. Fifty-seven patients underwent attempted LLR. The results concluded that LLR benefitted more by reducing the operating room time $(240 / 270$ mins, $\mathrm{p}=0.14)$, lower estimated blood loss $(250 / 500 \mathrm{ml}, \mathrm{p}<0.001)$, and shorter length of hospital stay $(5 / 6$ days, $\mathrm{p}<0.001)$. Median overall cost for LLR was lower at $\$ 11,376 \mathrm{vs}$. $\$ 12,523$ for OLR $(\mathrm{p}=0.077)$ [4].

Aldrighetti, et al. showed that Laparoscopic approach resulted in shorter operating time (150 min, P: 0.044) and lower blood loss (258 $\mathrm{ml}, \mathrm{P}:$ 0.008) with no difference in perioperative morbidity and mortality rate; laparoscopic approach was associated with a shorter hospital stay (6.3 days, P: 0.039) [5]. After a mean follows up of 32 months, disease-free survival and overall survival were 40.2 and 23.3 months for the laparoscopic group, and 47.7 and 31.4 months for OR group (PNS) [5]. In other studies looking specifically at LLS, the vast majority of these aforementioned clinical benefits were replicated $[1,2,4,6-12]$.

Figueredo and Yeung concluded that LLR reduced pain, inpatient days with more or less comparable operative outcome. Presence of skilled as well as an experienced surgical team, the operation would prove more safe and feasible [3]. Kawaguchi, et al. explained the cost for LLR were similar to those for OLR in a patient performing Left lateral sectionectomy along with a shorter period of hospitalization providing benefit to patient and hospital benefitted financially [13] (Table 3).

\begin{tabular}{|l|l|l|l|}
\hline Variables & LLR(N=102) & OLR(N=198) & P Value \\
\hline Operation time (min) & $214.5 \pm 8.078$ & $250.0 \pm 5.261$ & 0.0002 \\
\hline Blood Loss (ml) & $194.7 \pm 25.76$ & $398.9 \pm 26.41$ & $<0.0001$ \\
\hline Blood transfusion (ml) & $167.6 \pm 24.58$ & $423.6 \pm 30.85$ & $<0.0001$ \\
\hline Hospital stay(days) & $11.42 \pm 0.6191$ & $15.62 \pm 0.6572$ & $<0.0001$ \\
\hline ICU Stay (days) & $0.08824 \pm 0.0314$ & $0.3182 \pm 0.0401$ & 0.0002 \\
\hline
\end{tabular}

Table 3: Operative outcomes of patients who underwent Liver resections.

Cannon, et al. used deviation-based cost modeling and weighted average median cost to compare two procedures in order to determine laparoscopic group was slightly lower compared to open group. WAMC for the laparoscopic right hepatectomy group, however, was higher than the open group due to higher operation room cost [14].

Cleary, et al. concluded that the overall hospital costs for minor hepatic resections are less for LLR when compared to OLR [15]. Laparoscopy improved surgical and postsurgical outcomes because
Specific morbidity rates were similar but general morbidity rate was better in the laparoscopic group [16].

Medbery, et al. made a value-based analysis concluding that overall costs between LRH and ORH are equivalent, clinical outcomes after $\mathrm{LRH}$ are comparable to those after $\mathrm{ORH}$, supporting the value of laparoscopy in extensive right hepatic resections [10].

Polignano, et al. tried to explain the large-scale application of laparoscopic liver surgery could translate into significant savings to hospitals and health care program [17]. Financially, the total hospital costs of laparoscopic liver resection were either offset or improved because of a shorter length of stay, however, quick resumption of oral diet, less analgesic, and narcotic requirement, no difference in oncologic outcomes and other above mentioned clinical benefits superseded LLR to OLR. The vast majority of patients undergoing laparoscopic LLS for malignant disease continued to experience an uncomplicated postoperative course and was able to maintain a favorable cost-benefit over open surgery [12].

Cost parameter actually varied. Some authors depicted a reduction in cost for the laparoscopic approach. Some authors also illustrated more or less similar cost $[18,19]$. Laparoscopy can achieve short-term outcomes similar to those of open surgery for hepatocellular adenomas and has the additional benefits of a reduced blood loss, need for transfusion, and a shorter hospital stays [20-24]. Another important aspect of laparoscopic liver resection is aided not only by improved transection techniques, but also with advances in perioperative care, anesthesia, and post-operative care [25]. The Superficial PreCoagulation, Sealing, and Transection (SPST) method, a potentially "bloodless" and "ecofriendly" laparoscopic liver transection technique involving reusable devices: the VIO soft-coagulation system; VIO BiClamp (bipolar electrosurgical coagulation); Olympus SonoSurg (ultrasonic surgical system); and CUSA (ultrasonic aspirator).

Furthermore, we have reported the short-term outcomes of laparoscopic liver transection with the SPST method. The SPST method is a simple, efficient, and cost-effective surgical technique for laparoscopic liver resection. It is associated with low intraoperative blood loss and good short-term outcomes. We recommend that the SPST method should be used as a standard technique for laparoscopic liver resection [26].

Overall, our research not only supports the clinical efficacy of LLR to OLR but also cost-efficient. While not all laparoscopic approaches are equal, any minimally invasive approach may be an advance beyond the traditional open approach to clinical and economic aspects. Our analysis provides some evidence to prove that laparoscopic liver resection fulfills the clinical and economic aspects and to some extent has the potential to emerge as the standard approach for liver resection for selected patients. Efforts to reduce operative costs of LRH, while maintaining optimal patient outcomes should be the focus of surgeons and hospitals moving forward.

LLH is also a safe and effective treatment for selected patients with hepatolithiasis, with an advantage over OLH in the field of intraoperative blood loss, intraoperative transfusion, overall complication and postoperative recovery [27]. Enhanced Recovery After Surgery (ERAS) for laparoscopic liver resection is safe and effective. Patients in the ERAS group had a shorter hospital stay, fewer complications, and lower hospital costs [28]. The authors of other article tried to compare the perioperative outcomes of patients with primary hepatic carcinoma treated with Laparoscopic Hepatectomy $(\mathrm{LH})$ with those treated with Open Hepatectomy $(\mathrm{OH})$. Compared 
with $\mathrm{OH}, \mathrm{LH}$ could improve perioperative outcomes of primary hepatic carcinoma patients [29].

Some cases of Hepatic neoplasms required repeated operations. When we proceed with open approach repeated operation tends to be difficult for the patient as well as surgeons. Repeated more invasive approach around the neoplasm can promote metastasis as well increases painful days for the patient. In this scenario, the laparoscopic approach is rewarding as LLR may be preferred over open liver resection due to the well-known advantages of laparoscopy, such as reduced pain and improved possibility for repeated resections [30]. The technique of laparoscopic operation has advanced so much that these days laparoscopic liver resection in patients with a history of upper abdominal surgery is also feasible and safe [31].

Laparoscopic resection of HCC is feasible and safe in selected patients and can result in good surgical results, with similar outcomes in terms of overall and disease-free survival [5]. Laparoscopic approach for minor liver resection in elderly patients is safe and feasible with less blood loss, a shorter hospital stays, less postoperative complications and a better oncological outcome [32].

Benzing, et al. also studied performing laparoscopic hepatic resection in multimorbid elderly patients which supported the safety of operation even in those elderly patients [33]. Laparoscopic isolated caudate lobectomy remains a challenging procedure because of its deep location and proximity to the important vessels. The laparoscopic approach has been improvised with time in such a way that now it has become much convenient even for difficult liver resections like caudate lobectomy. The authors demonstrated the Arantius ligament suspension technique is a feasible and ideal method for a laparoscopic isolated caudate lobectomy for providing a rapid and safe left lateral lobe retraction [34].

LLR for HCC appears equally safe in cirrhotic and non-cirrhotic patients, and the advantages can be witnessed in those with advanced cirrhosis. Severe co-morbidities and conversion should be considered risk factors for complications-rather than the severity of cirrhosis and portal hypertension-when liver resection is performed laparoscopically. Such results may be of great interest to hepatic surgeons and hepatologists when deciding on the management of HCC within cirrhosis [35]. Another idea has also been advocated recently as Hybrid liver resection.

Coelho, et al. did a Propensity Score Matching Analysis to conclude that hybrid resection has better perioperative results than the open approach and is similar to pure laparoscopy. The authors insisted that the hybrid technique is considered a minimally invasive approach [36]. Patients with lesions in the posterosuperior (PS) segments of the liver have been considered poor candidates for laparoscopic liver resection (LLR).

D'Hondt, et al. conducted a case-matched study on Laparoscopic versus open parenchymal preserving liver resections in the posterosuperior segments. This study suggested that laparoscopic parenchymal preserving liver resections in the PS segments can be performed with comparable short-term outcomes as similar OLR [37].

Liver resection is an accepted treatment modality for large (greater than $5 \mathrm{~cm}$ ) Colorectal Liver Metastases (CLM). Laparoscopic resection appears to be a safe approach for resecting large CLM. Tumor size does not preclude laparoscopic hepatectomy [7]. LLR can be safely adopted today especially for lesions in the anterolateral segments. LLR for lesions in the difficult postero-superior segments and major liver resections especially in cirrhosis should only be attempted by surgeons who have acquired a minimum experience with 20 LLR [38].

In the pregnant lady, hepatocellular adenoma represents a significant diagnostic and therapeutic challenge. Anatomically favorable located lesions can be safely managed with laparoscopic liver resection. Laparoscopic liver resection should be considered as part of the currently available strategies for hepatocellular adenoma during pregnancy [39].

LLR in obese patients is also safe and effective [40]. The inflammatory response has been shown to be a major contributor to acute kidney injury. The laparoscopic technique, by decreasing the inflammatory response, may reduce the occurrence of postoperative acute kidney injury during liver resection surgery [41]. Laparoscopic resection in patients with CSPH can be performed safely in wellselected patients and expand the current surgical indications in patients with clinically significant portal hypertension [42]. Patients with a solitary HCC (diameter $5-10 \mathrm{~cm}$ ) who underwent open or laparoscopic liver resection were enrolled in a prospective observational study simultaneously analyzing the perioperative and follow up data. The authors came to a conclusion that laparoscopic liver resection is safe and feasible for the treatment of patients with large HCC [43]. Thus, a lot of evidence supports laparoscopic approach to open approach in liver resection.

Time has come now for the hospitals and doctors to make their patients aware of the advantages of both open as well as laparoscopic approach and simultaneously change the preferences for the choice of operative approach. The concepts and techniques in laparoscopic liver resection are totally different from those of open liver resection because of the different surgical views. Understanding the laparoscopy-specific concepts and techniques would facilitate safe and efficient execution of laparoscopic hepatectomy [44].

Limongelli, et al. performed a systematic review of the literature using the Medline, Embase, PubMed, and Cochrane databases to identify all studies published up to 2013 that compared laparoscopic and open liver (Laparoscopic Hepatic Resection (LLR) vs. Open Liver Resection (OLR)) and pancreatic (Laparoscopic Pancreatic Resection (LPR) vs. Open Pancreatic Resection) resection. This systematic review supports the economic advantage of laparoscopic over the open approach to the liver as well as pancreatic resection [45]. LLR is a practical replacement for OLR with probable advantages within the short-term outcomes for elected HCRM patients. Nevertheless, it remains an approach to advancement; in addition, randomized controlled trials and prolonged follow-up are necessary to verify its oncologic benefits and long-term survival [46].

Laparoscopic liver resection is safe and effective for well-selected patients with left IHD stones when performed by experienced surgeons. Laparoscopic liver resection resulted in shorter operation time and postoperative hospital stay, and a lower postoperative morbidity rate, than open hepatectomy [47]. Cirrhosis patient is always at risk during liver resection and looking for some published literature on this scenario comparing laparoscopic versus open approach. The authors of the review suggested that laparoscopic resection of hepatocellular carcinoma in patients with cirrhosis is safe and may provide improved patient outcomes when compared to the open technique [48].

Total laparoscopic anatomical S4, extended S4, or S4a or S4b segmentectomy is a feasible procedure for HCC. The Glissonian pedicle transection method is an effective technique for rapid and safe 
Page 6 of 7

control and subdivision of the S4 pedicle that facilitates anatomical laparoscopic S4, extended S4, and S4a and S4b segmentectomy [49]. Another theoretical advantage is that pneumoperitoneum pressure reduces hemorrhage from the hepatic vein [50]. Gustafson, et al. compared laparoscopic as well as open liver resection beyond the immediate postoperative period collecting morbidity data of thirty days and one year period. Their conclusion was that the advantages of laparoscopic liver resection may extend beyond the initial postoperative period, with fewer readmissions despite shorter hospital stays. This also may suggest lower long-term hospital costs [51]. OLR was also widely used for any kind of hepatic lesions. Different authors had different views in terms of cost but in terms of clinical benefits, almost all authors were in favor of laparoscopic approach. Excessive blood loss is associated with increased perioperative morbidity. Overall, our research not only supports the clinical efficacy of LLR to OLR but also cost-efficient. While not all laparoscopic approaches are equal, any minimally invasive approach may be an advance beyond the traditional open approach on clinical and economic fronts.

Laparoscopic liver resection can anytime be converted to open laparotomy if the anatomy is not clear or so as not to endanger patient safety. Our analysis provides some evidence to prove that laparoscopic liver resection fulfills the clinical and economic aspects of and to some extent has the potential to emerge as the standard approach for liver resection for selected patients.

\section{Conclusion}

LLR $(58740 \pm 1585 \mathrm{~N}=102)$ is not expensive compared to OLR $(61110 \pm 1964 \mathrm{~N}=198)$, with not much difference in terms of cost. The shorter length of hospital and ICU stay can guarantee a reduction in total cost reflecting indirect cost benefits. The clinical benefits are simultaneously reflected by lower blood loss, lower need for blood transfusion and lower complications rate for LLR compared to OLR. So, therefore, the new focus should be on reducing the operative costs in laparoscopic approach while maintaining the clinical efficacy.

\section{Limitations}

The infrastructures of primary hospitals and care centers, phobia for the expensive scenario as well as the scarcity of well-trained hepatic surgeons are the demerits that should also be taken into consideration. The concepts and techniques in laparoscopic liver resection are totally different from those of open liver resection because of the different surgical views and hence the acquisition of necessary skills is not that easy for open liver surgeons [44].

Our research was conducted in a well-equipped tertiary hospital of the 1st affiliated hospital of HMU of the fast developing country, China. The scenario should also be studied in other small hospitals too. Even though data on the technical feasibility of laparoscopic major resections and their benefits in terms of blood loss and functional recovery support the diffusion of minimally invasive approach, the limit of the technique is still represented by the reduced pool of suitable candidates [43]. This research included many different cases of liver resections because we wanted to include a greater number of patients. This research would have been more significant if we could study the same scenario in a single type of liver resection doing a casematched cohort study.

\section{Conflict of Interest}

The authors disclosed no potential conflicts of interest.

\section{References}

1. Kudo M (2014) Breakthroughs in the management of hepatocellular carcinoma: Celebrating 50 years of the liver cancer study group of Japan. Oncology 87(S1): 1-6.

2. Felekouras E, Kaparelos D, Papalambros E (2010) The history of liver surgery, hepatectomy and haemostasis. Hellenic J Surg 82: 280-296.

3. Figueredo EJ, Yeung RS (2008) Laparoscopic liver resection. Medscape J Med 10: 68-68.

4. Bhojani FD, Fox A, Pitzul K, Gallinger S, Wei A, et al. (2012) Clinical and economic comparison of laparoscopic to open liver resections using a 2to-1 matched pair analysis: an institutional experience. J Am Coll Surg 214: 184-195.

5. Aldrighetti L, Guzzetti E, Pulitano C, Cipriani F, Catena M, et al. (2010) Case-matched analysis of totally laparoscopic versus open liver resection for HCC: Short and middle term results. J Surg Oncol 102: 82-86.

6. Buell JF, Thomas MT, Rudich S, Marvin M, Nagubandi R, et al. (2008) Experience with more than 500 minimally invasive hepatic procedures. Ann Surg 248: 475-486.

7. Doughtie CA, Egger ME, Cannon RM, Martin RC, McMasters KM, et al. (2013) Laparoscopic hepatectomy is a safe and effective approach for resecting large colorectal liver metastases. Am Surg 79: 566-571.

8. Fretland AA, Kazaryan MA, Bjornbeth BA, Flatmark K, Anderson MH, et al. (2015) Open versus laparoscopic liver resection for colorectal liver metastases (the Oslo-CoMet Study): Study protocol for a randomized controlled trial. Trials 16: 73.

9. Martin RC, Mbah NA, St Hill R, Kooby D, Weber S, et al. (2015) Laparoscopic versus open hepatic resection for hepatocellular carcinoma: Improvement in outcomes and similar cost. World J Surg 39: 1519-1526.

10. Medbery RL, Chadid TS, Sweeney JF, Knechtle SJ, Kooby DA, et al. (2014) Laparoscopic versus open right hepatectomy: A value-based analysis. J Am Coll Surg 218: 929-939.

11. Murray A, Lourenco T, de Verteuil R, Hernandez R, Fraser C, et al. (2006) Clinical effectiveness and cost-effectiveness of laparoscopic surgery for colorectal cancer: Systematic reviews and economic evaluation. Health Technol Assess 10: 1-141.

12. Nguyen KT, Marsh JW, Tsung A, Steel JJ, Gamblin TC, et al. (2011) Comparative benefits of laparoscopic vs. open hepatic resection: A critical appraisal. Arch Surg 146: 348-356.

13. Kawaguchi Y, Otsuka Y, Kaneko H, Nagai M, Nomura Y, et al. (2016) Comparisons of financial and short-term outcomes between laparoscopic and open hepatectomy: Benefits for patients and hospitals. Surg today 46 : 535-542.

14. Cannon RM, Scoggins CR, Callender GG, Quillo A, McMasters KM, et al. (2013) Financial comparison of laparoscopic versus open hepatic resection using deviation-based cost modeling. Ann Surg Oncol 20: 2887-2892.

15. Cleary SP, Han HS, Yamamoto M, Wakabayashi G, Asbun HJ (2016) The comparative costs of laparoscopic and open liver resection: A report for the 2nd International consensus conference on laparoscopic liver resection. Surg Endosc 30: 4691-4696.

16. Dagher I, Di Gjuro G, Dubrez J, Lainas P, Smadja C, et al. (2009) Laparoscopic versus open right hepatectomy: A comparative study. Am J Surg 198: 173-177.

17. Polignano FM, Quyn AJ, de Figueiredo RS, Henderson NA, Kulli C, et al. (2008) Laparoscopic versus open liver segmentectomy: Prospective, casematched, intention-to-treat analysis of clinical outcomes and cost effectiveness. Surg Endosc 22: 2564-2570.

18. Lin NC, Nitta H, Wakabayashi G (2013) Laparoscopic major hepatectomy: A systematic literature review and comparison of 3 techniques. Ann Surg 257: 205-213. 
19. Vanounou T, Steel JL, Nguyen KT, Tsung A, Marsh JW, et al. (2010) Comparing the clinical and economic impact of laparoscopic versus open Liver resection. Ann Surg Oncol 17: 998-1009.

20. Landi F, De Angelis N, Scatton O, Vidal X, Ayav A, et al. (2017) Shortterm outcomes of laparoscopic vs. open liver resection for hepatocellular adenoma: A multicenter propensity score adjustment analysis by the AFC-HCA-2013 study group. Surg Endosc 31: 4136-4144.

21. Sotiropoulos GC, Prodromidou A, Machairas N (2017) Meta-analysis of laparoscopic vs. open liver resection for hepatocellular carcinoma: The European experience. J BUON 22: 1160-1171.

22. Ye MF, Xu GG, Gu JF, Zhou QL, Lin FQ, et al. (2017) Safety and efficacy evaluation of laparoscopy in colorectal cancer with liver metastasis. Eur Rev Med Pharmacol Sci 21: 27-32.

23. Noda T, Eguchi H, Wada H, Iwagami Y, Yamada D, et al. (2018) Shortterm surgical outcomes of minimally invasive repeat hepatectomy for recurrent liver cancer. Surg Endosc 32: 46-52.

24. Morise Z, Wakabayashi G (2017) First quarter century of laparoscopic liver resection. World J Gastroenterol 23: 3581-3588.

25. Aragon RJ, Solomon NL (2012) Techniques of hepatic resection. J Gastrointes Oncol 3: 28-40.

26. Itano O, Ikoma N, Takei H, Oshima G, Kitagawa Y (2015) The superficial precoagulation, sealing, and transection method: A "bloodless" and "ecofriendly" laparoscopic liver transection technique. Surg Laparosc Endosc Percutan Tech 25: e33-36.

27. Peng L, Xiao J, Liu Z, Zhu J, Wan R, et al. (2017) Laparoscopic left-sided hepatectomy for the treatment of hepatolithiasis: A comparative study with open approach. Int J Surg 40: 117-123.

28. Liang X, Ying H, Wang H, Xu H, Yu H, et al. (2016) Enhanced recovery program versus traditional care in laparoscopic hepatectomy. Medicine 95: e2835.

29. Jiang HT, Cao JY (2015) Impact of laparoscopic versus open hepatectomy on perioperative clinical outcomes of patients with primary hepatic carcinoma. Chinese Med Sci J 30: 80-83.

30. Aghayan DL, Kazaryan AM, Fretland AA, Sahakyan MA, Rosok BI, et al. (2017) Laparoscopic liver resection for metastatic melanoma. Surg Endosc 32: 1470-1477.

31. Ahn KS, Han HS, Yoon YS, Cho JY, Kim JH (2011) Laparoscopic liver resection in patients with a history of upper abdominal surgery. World J Surg 35: 1333-1339.

32. Badawy A, Seo S, Toda R, Fuji H, Fukumitsu K, et al. (2017) A propensity score-based analysis of laparoscopic liver resection for liver malignancies in elderly patients. J Invest Surg 17: 1-8.

33. Benzing C, Krenzien F, Gohlke D, Andreou A, Haber P, et al. (2017) Health-related quality of life after laparoscopic liver resection. J Minim Access Surg.

34. Chai S, Zhao J, Zhang Y, Xiang S, Zhang W (2017) Arantius ligament suspension: A novel technique for retraction of the left lateral lobe liver during laparoscopic isolated caudate lobectomy. J Laparoendosc Adv Surg Tech 28: 740-744.

35. Cipriani F, Fantini C, Ratti F, Lauro R, Tranchart H, et al. (2017) Laparoscopic liver resections for hepatocellular carcinoma. Can we extend the surgical indication in cirrhotic patients? Surg Endosc 32: 617-626.

36. Coelho FF, Kruger JAP, Jeismann VB, Fonseca GM (2017) Are hybrid liver resections truly minimally invasive? A propensity score matching analysis. J Laparoendosc Adv Surg Tech 27: 1236-1244.
37. D'Hondt M, Tamby E, Boscart I, Turcotte S, Parmentier I, et al. (2017) Laparoscopic versus open parenchymal preserving liver resections in the posterosuperior segments: A case-matched study. Surg Endosc 32: 1478-1485.

38. Goh BKP, Teo JY, Lee SY, Kam JH, Cheow PC, et al. (2017) Critical appraisal of the impact of individual surgeon experience on the outcomes of laparoscopic liver resection in the modern era: Collective experience of multiple surgeons at a single institution with 324 consecutive cases. Surg Endosc 32: 1802-1811.

39. Gryspeerdt F, Aerts R (2017) Laparoscopic liver resection for hemorrhagic hepatocellular adenoma in a pregnant patient. Acta Chir Belg 5: 1-4.

40. Ome Y, Hashida K, Yokota M, Nagahisa Y, Okabe M, et al. (2017) The safety and efficacy of laparoscopic hepatectomy in obese patients. Asian J Surg.

41. Moon YJ, Jun IG, Kim KH, Kim SO, Song JG, et al. (2017) Comparison of acute kidney injury between open and laparoscopic liver resection: Propensity score analysis. PloS One 12: e0186336.

42. Molina V, Sampson-Dávila J, Ferrer J, Fondevila C, Díaz Del Gobbo R, et al. (2017) Benefits of laparoscopic liver resection in patients with hepatocellular carcinoma and portal hypertension: A case-matched study. Surg Endosc 32: 2345-2354.

43. Ratti F, Cipriani F, Ariotti R, Giannone F, Paganelli M, et al. (2015) Laparoscopic major hepatectomies: Current trends and indications. A comparison with the open technique. Updates Surg 67: 157-167.

44. Ogiso S, Nomi T, Araki K, Conrad C, Hatano E, et al. (2015) Laparoscopy specific surgical concepts for hepatectomy based on the laparoscopic caudal view: A key to reboot surgeons' minds. Ann Surg Oncol 22: S327-333.

45. Limongelli P, Vitiello C, Belli A, Pai M, Tolone S, et al. (2014) Costs of laparoscopic and open liver and pancreatic resection: A systematic review. World J Gastroenterol 20: 17595-17602.

46. Qiu J, Chen S, Pankaj P, Wu H (2014) Laparoscopic hepatectomy is associated with considerably less morbidity and a long-term survival similar to that of the open procedure in patients with hepatic colorectal metastases. Surg Laparosc Endosc Percutan Tech 24: 517-522.

47. Shin YC, Jang JY, Kang MJ, Jung W, Chang Jet al. (2016) Comparison of laparoscopic versus open left-sided hepatectomy for intrahepatic duct stones. Surg Endosc 30: 259-265.

48. Twaij A, Pucher PH, Sodergren MH, Gall T, Darzi A, et al. (2014) Laparoscopic vs. open approach to resection of hepatocellular carcinoma in patients with known cirrhosis: Systematic review and meta-analysis. World J Gastroenterol 20: 8274-8281.

49. Kim YK, Han HS, Yoon YS, Cho JY, Lee W (2015) Total anatomical laparoscopic liver resection of segment 4 (S4), extended S4, and subsegments S4a and S4b for hepatocellular carcinoma. J Laparoendosc Adv Surg Tech 25: 375-379.

50. Wakabayashi G, Cherqui D, Geller DA, Han HS, Kaneko H, et al. (2014) Laparoscopic hepatectomy is theoretically better than open hepatectomy: Preparing for the 2nd International consensus conference on laparoscopic liver resection. J Hepato-biliary Pancreat Sci 21: 723-731.

51. Gustafson JD, Fox JP, Ouellette JR, Hellan M, Termuhlen P, et al. (2012) Open versus laparoscopic liver resection: Looking beyond the immediate postoperative period. Surg Endosc 26: 468-472. 